TecnoLógicas

ISSN-p 0123-7799

ISSN-e 2256-5337

Vol. 21, No. 42, pp. 231-244

Mayo-agosto de 2018

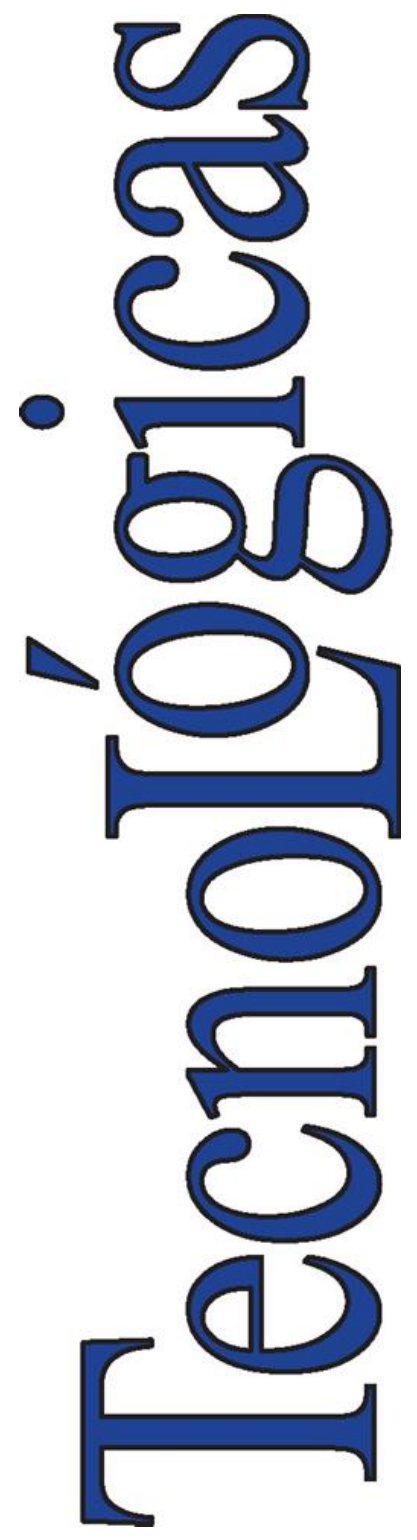

(C) Copyright 2015 por autores y Tecno Lógicas Este trabajo está licenciado bajo una Licencia Internacional Creative Commons Atribución (CC BY)

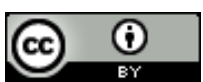

Artículo de Revisión/ Review Article

\section{Evaluación y aplicación de la incertidumbre de medición en la determinación de las emisiones de fuentes fijas: una revisión}

\section{Evaluation and implementation of measurement uncertainty for determining stationary source emissions: a review}

Jhon J. Cárdenas-Monsalve ${ }^{1}$, Andrés F. Ramírez-Barrera² y Edilson Delgado-Trejos ${ }^{3}$

Recibido: 19 de octubre de 2017

Aceptado: 18 de abril de 2018

Cómo citar / How to cite

J. J. Cárdenas-Monsalve, A. F. Ramírez-Barrera, y E. Delgado-Trejos, Evaluación y aplicación de la incertidumbre de medición en la determinación de las emisiones de fuentes fijas: una revisión. TecnoLógicas, vol. 21, no. 42, pp. 231-244, 2018.

1 Ingeniero Químico, Especialista en Salud Ocupacional, Instituto Tecnológico Metropolitano, Medellín-Colombia, jhoncardenas212235@correo.itm.edu.co

2 Bioingeniero, Magíster en Administración, Departamento Académico, Facultad de Ingenierías, Instituto Tecnológico Metropolitano, MedellínColombia, andresramirez@itm.edu.co

3 PhD Ingeniería LI Automática, MSc Automatización Industrial, Ingeniero Electrónico, Departamento de Calidad y Producción, Facultad de Ciencias Económicas y Administrativas, Instituto Tecnológico Metropolitano, Medellín-Colombia, edilsondelgado@itm.edu.co 


\title{
Resumen
}

Este artículo presenta una revisión de metodologías comúnmente citadas en la literatura para la estimación de la incertidumbre, como es la metodología no estocástica de la Guía para la Expresión de la Incertidumbre de Medida (GUM), la cual provee una estructura de estimación con limitaciones en su implementación, como son: cálculo de derivadas parciales, suposición de linealidad de los modelos e identificación de las fuentes de incertidumbre y sus distribuciones de probabilidad. Por otro lado, se discuten otros métodos para estimar la incertidumbre, como son: Monte Carlo, Conjuntos Difusos, Intervalo Generalizado, Inferencia Bayesiana, Caos Polinomial y Bootstrap, que a diferencia de la GUM, presentan limitaciones de costo computacional y requieren de conocimientos más especializados para su implementación. El objetivo de este artículo es reportar el grado de aplicación y difusión de los métodos de estimación de la incertidumbre en las emisiones de fuentes fijas, encontrándose que la mayoría se enfoca en estudios usados para la elaboración de inventarios de gases de efecto invernadero (GHG), y son escasos los orientados a la medición de las emisiones de fuentes fijas usando monitoreos de lectura directa, como también los métodos definidos por la Agencia de Protección Ambiental de los Estados Unidos (US EPA). Se discute finalmente las fortalezas y debilidades que dan lugar al fomento de nuevas investigaciones en esta área del conocimiento.

\section{Palabras clave}

Incertidumbre de medición, Guía para la Expresión de la Incertidumbre de Medida GUM, Método Monte Carlo, métodos estocásticos.

\begin{abstract}
This paper presents a review of commonly-cited methods for estimating uncertainty in the literature. One of them is the non-stochastic approach proposed by the Guide to the Expression of Uncertainty in Measurement (GUM), which provides an estimation framework with limitations for the implementation, such as computation of partial derivatives, linear model assumptions, and uncertainty source identification with probability distributions. Other methods to estimate uncertainty are discussed as well; they include Monte Carlo, Fuzzy Sets, Generalized Intervals, Bayesian Inference, Polynomial Chaos, and Bootstrap, which in contrast to GUM present limitations regarding computational cost and require more specialized knowledge to be implemented. The aim of this work is to report the level of application and dissemination of methods for estimating the uncertainty of emissions caused by stationary sources. Most of the works in this field were found to be focused on the creation of inventories of Greenhouse Gases (GHG), and very few of them on the uncertainty associated with measuring the emissions of stationary sources using direct reading monitoring or those defined by the Environmental Protection Agency of the United States (US EPA). Finally, strengths and weaknesses are discussed in order to promote new research in this knowledge area.
\end{abstract}

\section{Keywords}

Measurement uncertainty, Guide to the Expression of Uncertainty in Measurement GUM, Monte Carlo method, stochastic methods. 


\section{INTRODUCCIÓN}

La comunidad científica ha trabajado intensamente durante décadas tratando de entender y formalizar la incertidumbre. La mayor incertidumbre en un problema es lo menos preciso o correcto que se pueda estar del entendimiento de este, es así que muchos analistas están de acuerdo en que se debería entender y evaluar la incertidumbre que existe en la solución de problemas, o hacer predicciones, tratando de buscar un punto de equilibrio entre la incertidumbre y la precisión que se busca [1]. La incertidumbre de una medición se define como un parámetro no negativo que caracteriza la dispersión de los valores atribuidos a un mensurando, a partir de la información utilizada, y dependiendo de cómo esta dispersión es caracterizada, incluye componentes de efectos sistemáticos, tales como los asociados a correcciones $\mathrm{y}$ valores asignados a patrones [2].

En cuanto al medio ambiente, las implicaciones de la incertidumbre son particularmente relevantes en la evaluación de las posibles opciones de regulación, como es el seguimiento y control de los niveles de calidad del aire [3]. Actualmente, con el incremento de la aplicación de la metrología en las mediciones ambientales, se han explorado diferentes metodologías para la estimación de la incertidumbre, entre las cuales se incluye la aplicación de la Guía para la Expresión de la Incertidumbre de Medida (GUM), la cual se ha derivado de una metodología no estocástica [4]. Esta guía ha sido ampliamente aceptada dentro de la comunidad científica desde su primera versión de 1993 y para el 2017 permanece vigente la versión del año 2008 [5]. La GUM cuenta, además, con dos complementos para su aplicación. El primero es una introducción con documentos relacionados [6]; y el segundo es un suplemento que permite trabajar con modelos multivariados y cualquier número de variables de salida [7]. La complejidad de los modelos matemáticos requerida para estimar la incertidumbre asociada con los inventarios de emisiones para fuentes fijas, como también el seguimiento dinámico a esas emisiones, ha limitado la aplicación de la GUM, si bien, son frecuentes aplicaciones de esta [8], [9], [10], es más común encontrar trabajos usando otros métodos como Monte Carlo, Bootstrap, Intervalo Generalizado, Conjuntos Difusos, entre otros [3], [11], [12], [13], [14], [15], [16]. Las emisiones contaminantes de las fuentes fijas pueden ser monitoreadas de forma continua [17] o mediante los métodos definidos por la Agencia de Protección Ambiental de los Estados Unidos (US EPA) [18], estos últimos adoptados en Colombia mediante la Resolución 0935 de 2011 por el Instituto de Hidrología, Meteorología y Estudios Ambientales [19]. Ambas formas de monitoreo requieren de la estimación de la incertidumbre asociada al proceso de medición con el fin de garantizar la trazabilidad y validez de los datos generados, para así poder usarlos en la verificación de cumplimientos legales y elaboración de inventarios de emisiones. Dentro de la literatura revisada sobre la determinación de la incertidumbre asociada a las emisiones en fuentes fijas, resalta el trabajo desarrollado por [20], donde la guía GUM es aplicada para la estimación de la incertidumbre asociada al monitoreo de Óxidos de Nitrógeno (NOx), usando el método US EPA 7 [20].

En este artículo, se realiza una revisión del estado del arte en la evaluación de la incertidumbre de medición, se exploran los diferentes métodos y metodologías disponibles para su estimación, y el grado de aplicación de las mismas al campo de las mediciones de las emisiones de fuentes fijas, para lo cual se inicia con una revisión sobre las metodologías disponibles para la estimación de la incertidumbre y se termina con los hallazgos de los estudios realizados aplicados al área de interés del estudio. Además, la revisión destaca las fortalezas, debilidades, grado de difusión y apli- 
cación de cada una de las metodologías que estiman la incertidumbre.

\section{ESTIMACIÓN DE LA INCERTIDUMBRE: METODOLOGÍAS Y APLICACIONES}

En la Fig. 1, se muestra un esquema general de los métodos para la estimación de la incertidumbre, y el uso de estos en la cuantificación de la incertidumbre asociada a la determinación de las emisiones desde fuentes fijas.

Dentro de la variabilidad de propuestas para la estimación de la incertidumbre, se encuentran herramientas como la GUM [5], Conjuntos Difusos [12], Caos Polinomial [13], Remuestreo (Bootstrap) [14], Inferencia Bayesiana [15], Intervalo Gene- ralizado [16] y Monte Carlo (MCM) [21]. La combinación de la GUM con otros métodos de estimación, se ha empleado principalmente en aquellos casos donde los modelos matemáticos son muy complejos y la aplicación de la guía GUM resulta difícil de implementar, debido a la imposibilidad de realizar una identificación exhaustiva de todas las fuentes de incertidumbre y sus respectivas distribuciones estadísticas asociadas, lo que conduce a que se deban realizar simplificaciones del modelo de propagación de la incertidumbre [22]. Una ventaja del uso de ambas metodologías de forma simultánea, es que permite validar las simplificaciones realizadas en el proceso de estimación de la incertidumbre al aplicar la guía GUM.

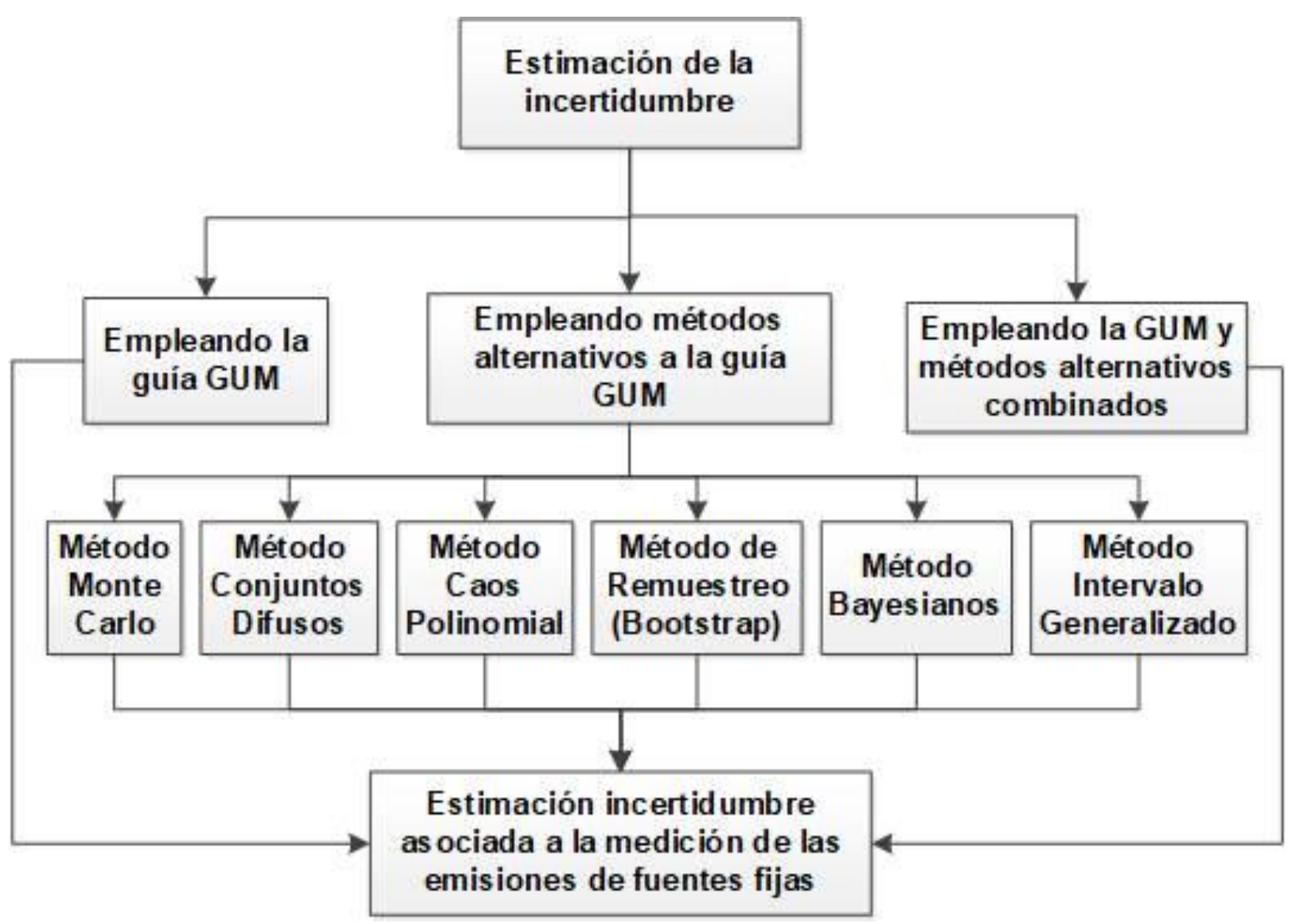

Fig. 1. Esquema de los métodos para estimación de la incertidumbre. Fuente: autores. 


\subsection{Estimación de la incertidumbre aplican- do la guía GUM y documentos relaciona- dos}

La evaluación de la incertidumbre puede hacerse por métodos como el propuesto en los documentos desarrollados por el Joint Committee for Guides in Metrology (JCGM), entre los documentos desarrollados está la guía GUM [5], la cual está compuesta por un documento introductorio y otros reportes relacionados [6], además del suplemento 2, el cual permite trabajar con modelos multivariados en relación con cualquier número de variables de salida o múltiples mesurandos [7]. La guía GUM desde su primera versión publicada en 1993, estableció una serie de recomendaciones con gran acogida por la comunidad científica, lo que llevó a que se presentaran numerosas aplicaciones de esta en diferentes campos del conocimiento, entre los que se incluye: implementación de un procedimiento para la incertidumbre combinada en mediciones de espectrometría gamma [23], mediciones analíticas a través el desarrollo de la guía para la cuantificación de la incertidumbre [24], evaluación y discusión de la estimación de la incertidumbre en análisis farmacéuticos [25], entre otros.

En la primera línea de la Fig. 2, se presentan las guías propuestas por el JCGM para la aplicación de la guía GUM, así como un recuento de algunas publicaciones que han usado esta guía para estimar la incertidumbre de medición, mientras en la segunda y tercera línea, se hace mención de aquellos trabajos que buscan cubrir aspectos necesarios para la estimación de la incertidumbre y que la GUM no contempla o incluye para su implementación.

Cuando la estimación de la incertidumbre se realiza mediante el uso de distribuciones de probabilidad, se identifican dentro de la literatura diferentes trabajos que abordan vacíos que posee la GUM para su implementación, entre los cuales están: evaluación de la incertidumbre en medi- ciones, problemas y herramientas [4]; evaluación de la incertidumbre para múltiples mediciones usando GUM [26]; evaluación de la incertidumbre para múltiples mediciones usando GUM, parte II [27]; exactitud de mediciones simples, las cuales no son mencionadas por la GUM [28]; aplicación para el chequeo de consistencia en la evaluación de la incertidumbre en mediciones de múltiples réplicas [29]; de la GUM a métodos alternativos para la evaluación de la incertidumbre de medición [30]; evaluación de la incertidumbre para múltiples mediciones por GUM, parte III, usando coeficientes de correlación [31].

De otra parte, [32], discuten el problema de la cuantificación del error sistemático sobre la medición de la incertidumbre total, eludiendo los conceptos de grados de libertad y probabilidad subjetiva que hacen parte de la razón de ser de la GUM, aplicados al caso cuando la función de medición es fuertemente no-lineal y el uso de la aproximación lineal puede ser inadecuada, donde los términos de orden superior de la serie de Taylor no pueden ser despreciados, para lo cual se propone el uso de expresiones generalizadas para aquellos términos de segundo y tercer orden en la evaluación de la incertidumbre estándar [33], mientras en el trabajo desarrollado por [34], se realiza una revisión de las debilidades de la guía GUM cuando se efectúan mediciones con resultados cercanos a cero.

Por otro lado, se encuentran publicaciones como las mostradas en la cuarta línea de la Fig. 2, encaminadas a extender la implementación de la GUM, tal es el caso del trabajo propuesto por [35], en el cual se estima la incertidumbre de medición mediante el desarrollo de una aplicación que emplea el software comercial (Maple) y que permite trabajar con funciones simbólicas, caso similar al mostrado por [36], donde se pone en evidencia las ventajas del cálculo simbólico frente al numérico para la estimación de las derivadas parciales, cuando se emplean diferentes paquetes comerciales. 
Evaluación y aplicación de la incertidumbre de medición en la determinación de las emisiones de fuentes fijas:

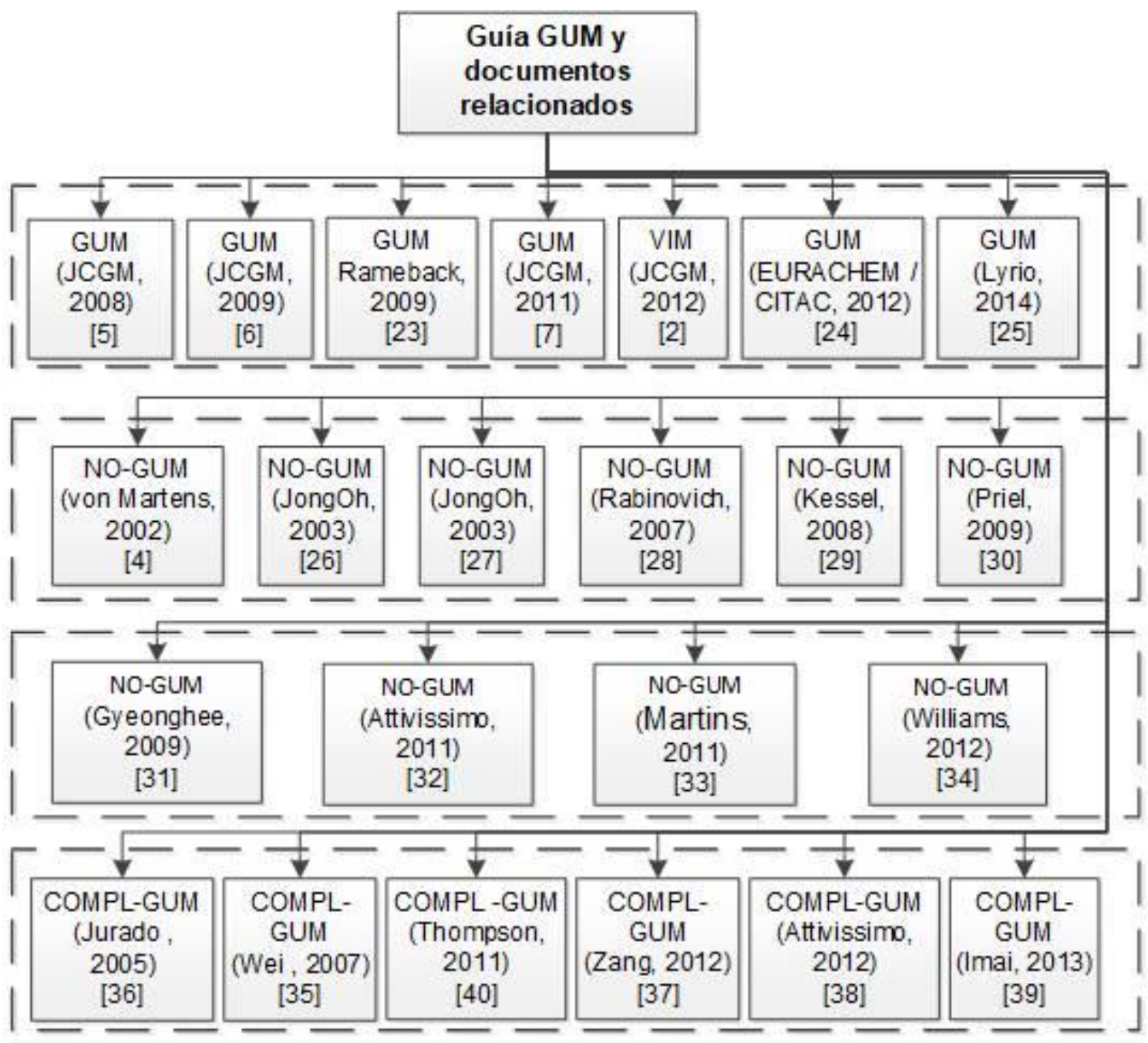

GUM: Guía para la estimación de la incertidumbre de medida.

VIM: Vocabulario Internacional de Metrología.

COMPL - GUM: Trabajos que sirven como complemento para la aplicación de la guía GUM.

NO - GUM: Aplicación de métodos no estocásticos a la estimación de la incertidumbre no incluidos en la GUM

Fig. 2. Aplicación de la GUM y documentos relacionados en la estimación de la incertidumbre. Fuente: autores.

Por otro lado, trabajos como el de Zang [37] buscan ayudar a la interpretación de la GUM y resumen las terminologías de probabilidad pertinentes para la cuantificación de la incertidumbre en ingeniería, mientras [38] presentan ideas y observaciones sobre el uso del frecuentismo y la aplicación de la inferencia bayesiana en la estimación de la incertidumbre, en térmi- nos de méritos y fallas de la aplicación bayesiana comparada con la frecuentista. El trabajo desarrollado por [39] describe la manera de obtener mediciones confiables, usando la expresión de trazabilidad metrológica junto con la medición de incertidumbre. Las deficiencias de la metodología GUM, son expuestas y presentadas bajo el término de incertidumbre oscura por [40], 
la cual se refiere a aquella incertidumbre que aparece como una dispersión grande e inesperada de los valores, y examina la evidencia de su ocurrencia al comparar los resultados obtenidos por la GUM frente a los arrojados por la reproducibilidad estimada a partir de las comparaciones entre los diferentes laboratorios. Los resultados muestran una tendencia general en la cual los valores de incertidumbre estándar estimados empleando la guía GUM son sustancialmente inferiores a la desviación estándar de reproducibilidad, lo que evidencia problemas de implementación de la GUM al no incluir todas las fuentes de incertidumbre en el modelo de propagación.

\subsection{Métodos alternativos a la GUM para la estimación de la incertidumbre}

La metodología basada en la GUM por derivadas parciales presenta varias limitaciones, en primer lugar, implica el cálculo de derivadas de primer orden para cada componente de la magnitud de salida, requiriéndose un considerable procesamiento matemático especializado, que puede llegar a ser exhaustivo si el modelo matemático es complejo; y en segundo lugar, esta no tiene la posibilidad de hacer predicciones exactas sobre la distribución de probabilidad de la magnitud de salida, si las magnitudes de entrada no tienen distribución normal [41], y es bien conocido que el conocimiento de la distribución de probabilidad es esencial para determinar el intervalo de cobertura.

La Fig. 3 muestra el diagrama conceptual sobre la revisión de diferentes trabajos basados en otros métodos para la estimación de la incertidumbre. Entre los métodos revisados el de mayor difusión y aceptación es el Método Monte Carlo, el cual realiza un muestreo aleatorio de las distribuciones de probabilidad de las magnitudes de entrada; por lo tanto, no requiere calcular derivadas de primer orden, ni la función de densidad de probabilidad de la magnitud de salida. En el suplemento 1 del JCGM para estimación de la incertidumbre usando el MCM, se dan condiciones específicas sobre cuándo es aplicable el método GUM a sistemas lineales y no lineales y en qué casos es preferible el uso de una herramienta como MCM [42].

El MCM se utilizó para la estimación de la incertidumbre por [42], quienes describen la situación actual de la evaluación de la incertidumbre en el contexto de las normas internacionales, y se recomienda una aplicación usando Monte Carlo para la evaluación de la incertidumbre de medición basada en la propagación de distribuciones. El método Monte Carlo ha sido también empleado para estimar la incertidumbre en modelos fuertemente no lineales [43], y ha demostrado ser una herramienta efectiva y versátil para la determinación de funciones de distribuciones de probabilidad ( $p d f$ ) para mesurandos [44]. Adicionalmente, se han desarrollado algoritmos que permiten al usuario elegir la distribución de salida preferida para el mesurando [45], más que la asignada implícitamente en el suplemento 1 de la GUM, esto unido a investigaciones que buscan mejorar la eficiencia de muestreo y convergencia del método Monte Carlo [46], con el fin de reducir el gasto computacional tanto como sea posible.

Para la estimación de la incertidumbre existen otros métodos, como es Caos Polinomial, el cual es más apropiado para modelos a gran escala, donde el método Monte Carlo converge lentamente [13], o en modelos de alta complejidad como ocurre con las ratas de reacción al interior de un reactor [47]. El método Bootstrap ha sido utilizado para aquellos casos donde el método Monte Carlo no puede ser aplicado directamente para la caracterización de la incertidumbre, como es el caso de ensayos donde no se puede medir directamente la magnitud de interés [14], asimismo este método se ha empleado para la estimación de la incertidumbre en la inferencia de los estimativos de las reservas de petróleo y gas [48]. 
Evaluación y aplicación de la incertidumbre de medición en la determinación de las emisiones de fuentes fijas: una revisión
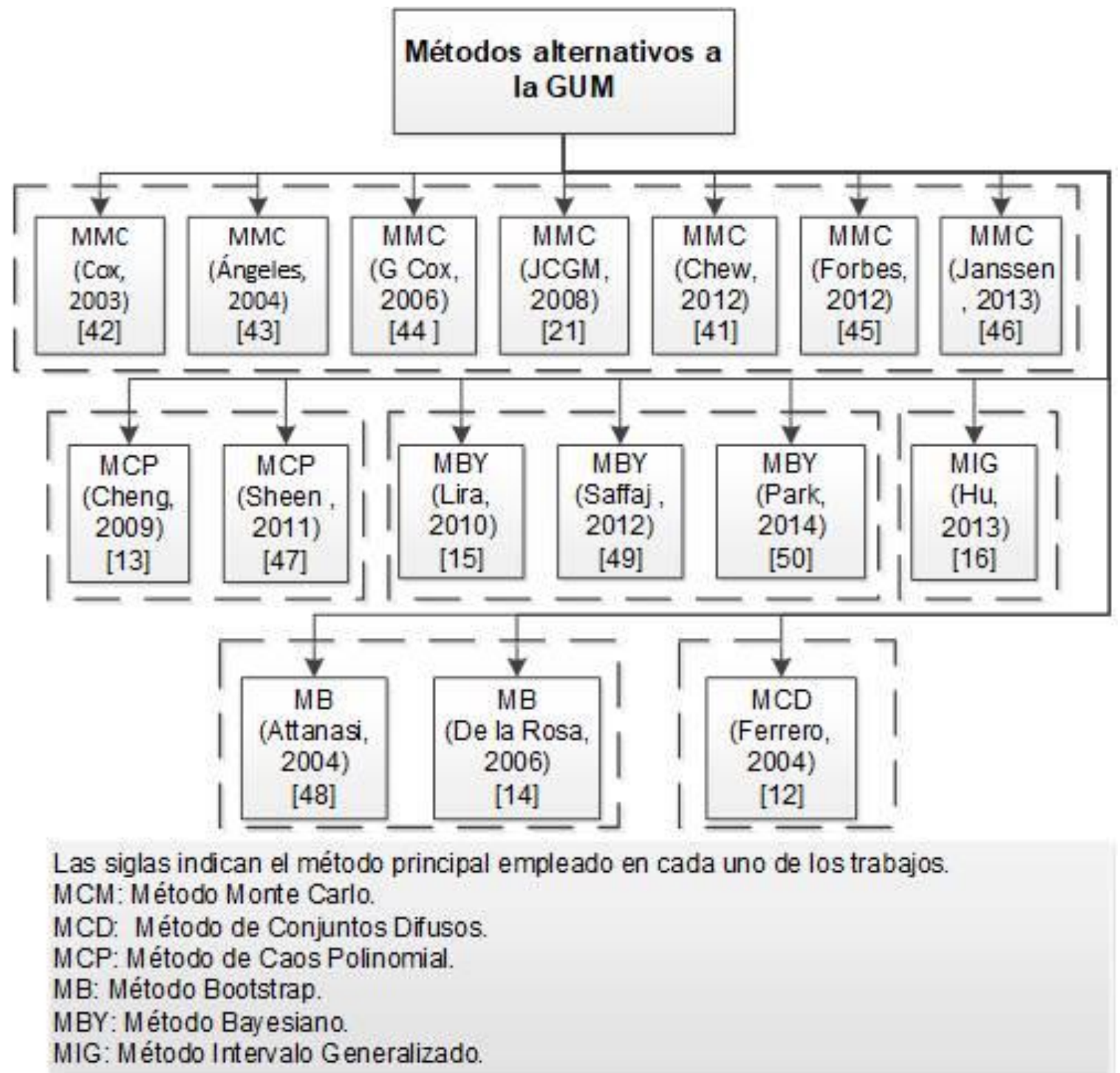

Fig. 3. Aplicación de métodos alternativos a la GUM en la estimación de la incertidumbre. Fuente: autores.

Otro método es la Inferencia Bayesiana, por medio de la cual se ha demostrado [15] que hay maneras alternativas de aplicar el teorema de Bayes y que estas formulaciones alternativas producen los mismos resultados. El método bayesiano comparado con el método clásico (GUM), es más corto y no requiere conocer todas las etapas individuales en los cuales un proceso puede ser descompuesto (ejemplo: aplicaciones de métodos analíticos o bio-analíticos), ya que es tomado como un todo [49]. El método bayesiano también ha mostrado sus bondades en la cuantificación de la incertidumbre asociada a la selección del mejor modelo [50], lo cual puede ser problemático debido a la posible subestimación de la variabilidad de las predicciones. Por último, se referencian otros dos métodos que han sido empleados en la estimación de la incertidumbre: los Conjuntos Difusos [12] y el Intervalo Generalizado [16]. De todos los métodos alternativos a la GUM, el que cuenta con mayor difusión y aplicabilidad es Monte Carlo, al punto que el JCGM emitió en 2008 una guía con el fin de ayudar en su implementación [21]. En general, se encuentra que muchos de los esfuerzos investigativos sobre el uso de métodos alternativos a la GUM para estimar la 
incertidumbre, se centran en la obtención de un método estadístico estable que permita una muy buena determinación de la incertidumbre de los procesos de medición, que paralelamente disminuya el porcentaje de duda en los cálculos matemáticos y permita crear un modelo que se acerque al proceso de medición real, tomando todas las fuentes involucradas en el proceso.

\subsection{Estimación de la incertidumbre em- pleando métodos combinados}

Por otro lado, se encuentran los estudios en los cuales se ha utilizado la combinación de diferentes métodos de estimación, como los que se muestran en la Fig. 4.

La aplicación de métodos híbridos a la estimación de la incertidumbre es amplia y comprende diferentes campos del conocimiento, entre los que se incluyen trabajos donde se aplica MCM y la guía GUM como son: estimación de la incertidumbre en pruebas de compatibilidad electromagnéti- ca [22], análisis de cadmio por GFAAS [51], medición del poder calórico de combustión de un combustible usando una bomba calorimétrica [52], y mediciones de resistencia y potencia eléctrica [53], asimismo, se encuentran trabajos como el desarrollado por Torres [54], donde se usan ambas metodologías de estimación en la cuantificación de la incertidumbre asociada a la determinación de la concentración de sólidos totales, mientras [55], describe las propiedades y limitaciones de la estructura para estimar la incertidumbre de la GUM y el método Monte Carlo, empleando un modelo de razones. De forma similar, una aplicación para la estimación de la incertidumbre basada en la teoría del Caos Polinomial es propuesta por [56] y se compara con el método tradicional basado en la guía GUM. Por último, [1] desarrollan un panorama de la evolución de la estimación de la incertidumbre, tanto desde el punto de vista estocástico como no estocástico.

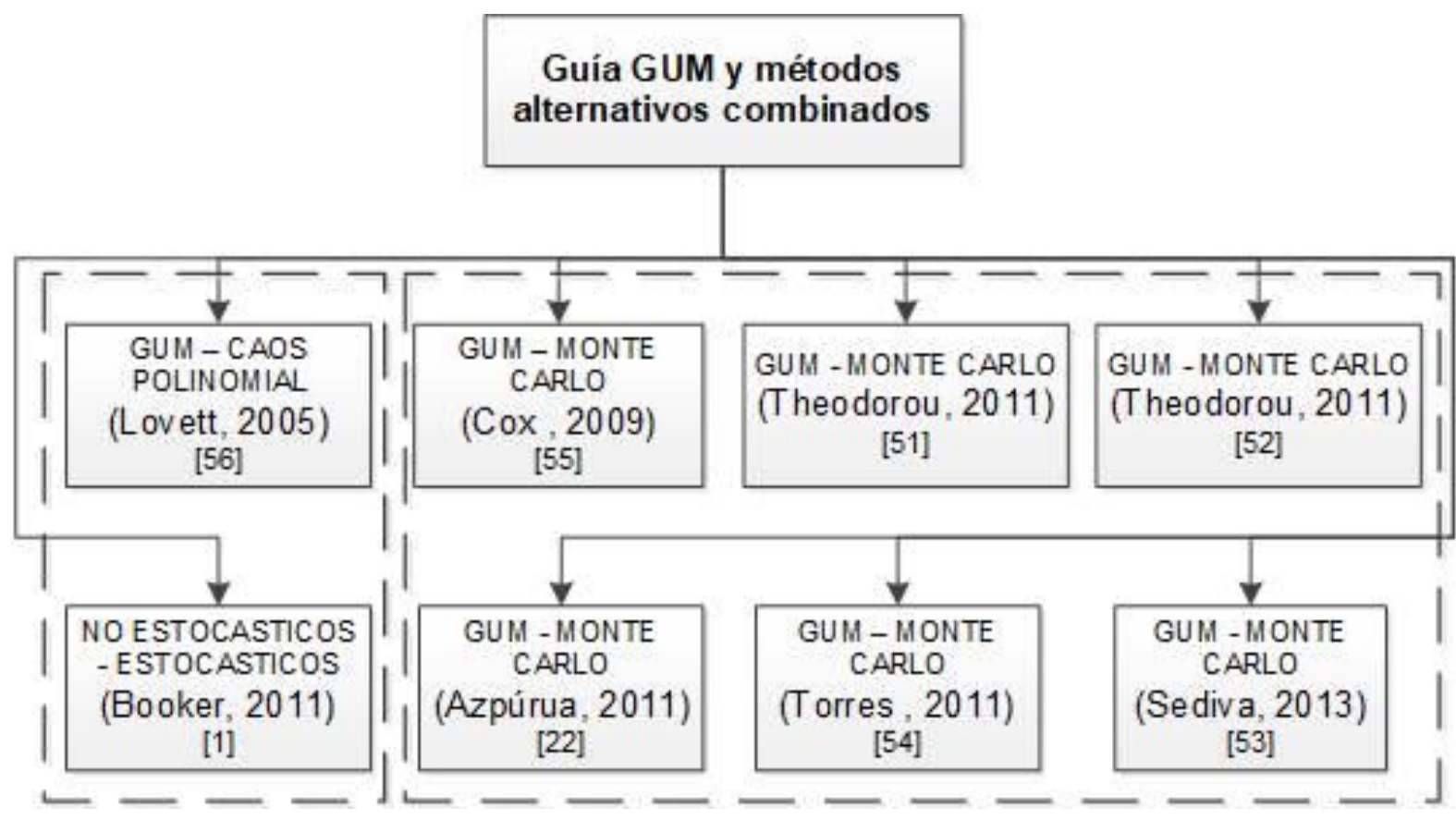

Fig. 4. Aplicación de la GUM y métodos alternativos combinados a la estimación de la incertidumbre. Fuente: autores. 
Evaluación y aplicación de la incertidumbre de medición en la determinación de las emisiones de fuentes fijas: una revisión

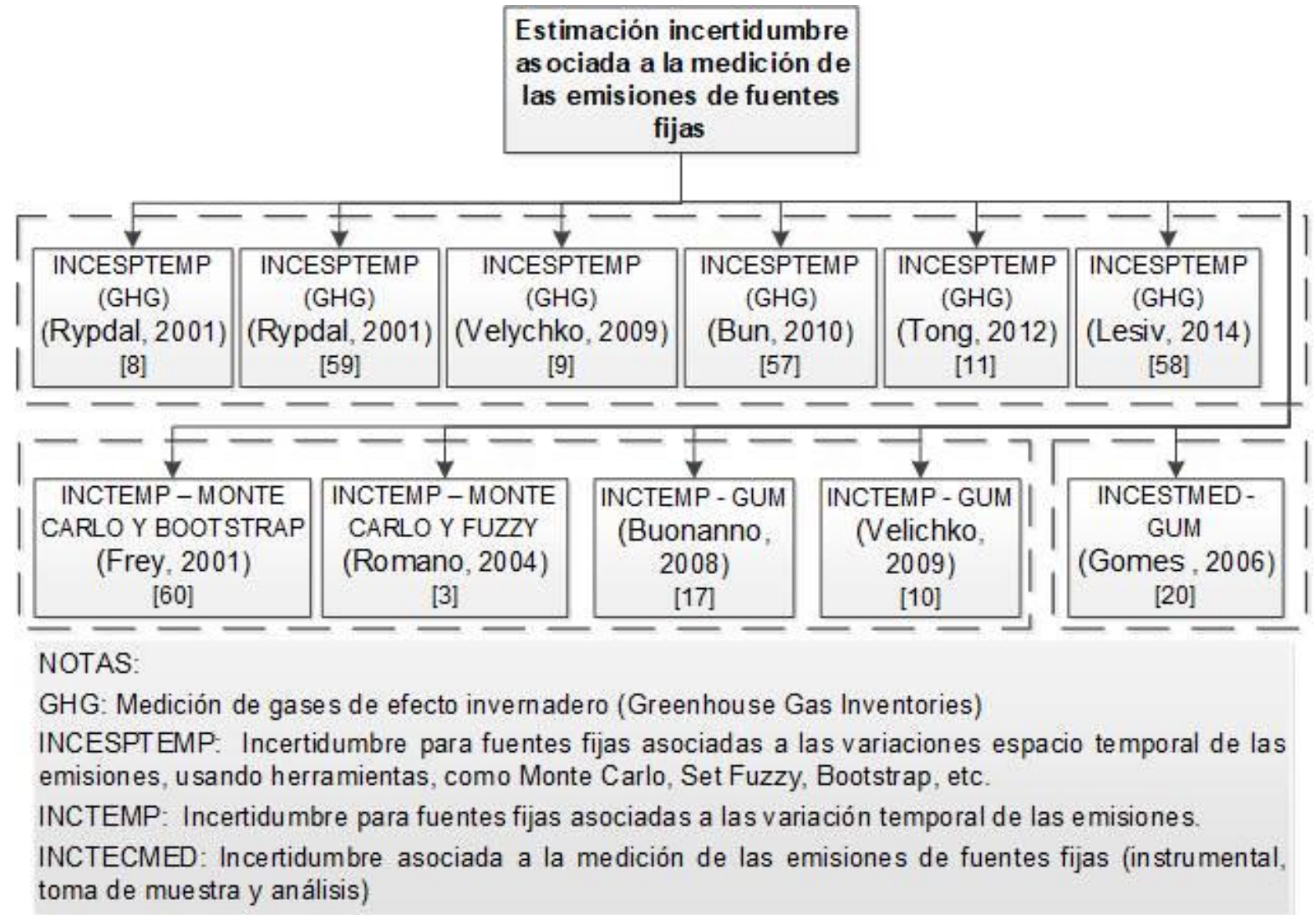

Fig. 5. Cuantificación de la incertidumbre en fuentes fijas mediante el uso de diferentes métodos. Fuente: autores.

\subsection{Métodos empleados a la estimación de la incertidumbre en fuentes fijas}

Una vez completada la revisión de las metodologías para estimación de la incertidumbre y sus aplicaciones en diferentes campos, la revisión se centra en identificar aquellos trabajos donde se han empleado diferentes herramientas, tanto de carácter estocástico como no estocástico, en la estimación de la incertidumbre asociada a las emisiones de fuentes fijas. Dentro de estos trabajos se resaltan los que se presentan en las líneas primera y segunda de la Fig. 5 , y que se pueden clasificar en dos partes, la primera comprende los trabajos identificados con las siglas INCESPTEMP e INCTEMP, que se orientan a estimar la variación espacio temporal de las fuentes y la incertidumbre asociada, dentro de esta categoría se encuentra una amplia cantidad de estudios en la literatura, entre los que se incluyen los realizados para la ela- boración de los inventarios de gases de efecto invernadero (GHG) [8], [9], [11], [57], [58], [59], así como trabajos donde se estima la variación temporal mediante la recolección de datos de mediciones continuas, los cuales se pueden usar para cuantificar la incertidumbre asociada mediante métodos como Monte Carlo, Bootstrap y Conjuntos Difusos [3], [60]. Por otra parte, la segunda corresponde a los trabajos identificados con la sigla INCESTMED, usados para estimar la incertidumbre asociada a la medición instrumental de la emisión desde fuentes fijas, empleando los métodos definidos en el Código Federal de Regulaciones (CFR), título 40 parte 60 Apéndices A1 a A8 [18], el cual incluye métodos para la determinación de Material Particulado (MP), Óxidos de Nitrógeno $\left(\mathrm{NO}_{\mathrm{x}}\right)$, Dióxido de Azufre $\left(\mathrm{SO}_{2}\right)$ y Neblinas Ácidas $\left(\mathrm{H}_{2} \mathrm{SO}_{4}\right)$, entre otros. $\mathrm{Al}$ respecto son escasas las publicaciones en la literatura, de tal manera que esta revisión solo logró identificar el 
trabajo desarrollado por [20], donde se aplica la GUM para la estimación de la incertidumbre asociada a la medición de las emisiones de $\mathrm{NO}_{\mathrm{x}}$, cuando la muestra es colectada de acuerdo con lo descrito en el método EPA 7 "Determinación de emisiones de óxidos de nitrógeno de fuentes fijas".

\section{CONCLUSIONES Y RECOMENDACIONES}

Son pocas las publicaciones en la literatura referidas a metodologías específicas que permitan estimar la incertidumbre asociada al procedimiento instrumental empleado para la determinación de las emisiones de fuentes fijas, cuando se aplican los métodos 1 al 8 propuestos por la Agencia de Protección Ambiental de los Estados Unidos (US EPA). Esta revisión encontró que, entre los pocos trabajos en esta área, se encuentra el reportado en [20], el cual solo se limita a la estimación de la incertidumbre para el método EPA 7 mediante la aplicación de la guía GUM, sin cubrir los métodos restantes y la posibilidad de implementar métodos de estimación alternativos.

La revisión permitió, además, identificar que la aplicación de la metodología de estimación propuesta en la guía GUM, a la estimación de la incertidumbre asociada a la medición de fuentes fijas, requiere superar los siguientes inconvenientes: 1) Incremento de complejidad cuando el método de medición comprende muchas etapas y cada una contribuye a la incertidumbre del resultado final; 2) Conocer con exactitud la distribución estadística asociada a cada fuente, con el fin de poder reducirla e ingresarla al modelo de propagación en el que se basa la GUM; 3) Suponer un comportamiento lineal de la función de medición, aunque hay casos en los cuales esta es fuertemente no-lineal y el uso de la aproximación lineal puede ser inadecuada, dado que los términos de orden superior de la serie de Taylor no pueden ser despreciados; 4) Calcular derivadas de primer orden de cada componente de la magnitud de salida, lo cual demanda buen entendimiento y procesamiento matemático y puede ser agotador si el modelo matemático es complejo; 5) Predecir la distribución de probabilidad de la magnitud de salida con precisión, cuando las magnitudes de entrada no poseen distribución normal; y 6) La imposibilidad de ingresar al modelo todas las fuentes de incertidumbre, ya sea porque se desconoce su presencia, o no se cuenta con un estimativo de su influencia.

De otra parte, se encuentran los métodos alternativos que a diferencia de la metodología basada en la GUM no poseen todas las limitaciones anteriores, pero en cambio presentan inconvenientes, como: 1) Incremento de los costos computacionales, que puede convertirse en un factor limitante para su implementación; 2) Algunos métodos estocásticos, como Monte Carlo requieren de la selección apropiada de la distribución estadística para los parámetros del modelo, lo cual puede ser un inconveniente debido a la falta de compresión subyacente de los fenómenos físicos; y 3) El grado de conocimiento requerido para la selección e implementación del método más apropiado a usarse, exige un alto grado de experticia, cuando se compara con el requerido para el empleo de la guía GUM.

La aplicación en simultáneo de ambas metodologías, GUM y métodos alternativos, para la estimación de la incertidumbre, ha permitido una validación de los resultados obtenidos cuando los modelos matemáticos que se trabajan son complejos y la identificación de todas las fuentes de incertidumbre puede convertirse en un procedimiento extenuante, de forma que la aplicación de la metodología basada en la guía GUM tiende a subestimar el valor de la incertidumbre, y el uso en simultáneo con otro método permite su validación.

La necesidad de cuantificar la incertidumbre radica en el papel que juega en las mediciones y en su importancia como he- 
rramienta para dar solución al problema que se presenta cuando se requiere la toma de decisiones que involucran límites de carácter regulatorio, o de conformidad, como es el caso de las mediciones de las emisiones de fuentes fijas. Es por esto, que se requieren nuevos estudios para el desarrollo de una metodología conjunta que implemente métodos estocásticos y no estocásticos, para la determinación de la incertidumbre asociada a la determinación de las emisiones de este tipo de evaluaciones, con el fin de alcanzar un mejoramiento importante en el proceso de medición de fuentes fijas.

\section{AGRADECIMIENTOS}

Este artículo se presenta en el marco de la tesis de Maestría en Automatización y Control denominada: "Metodología para la determinación de la incertidumbre asociada a la medición en fuentes fijas usando la guía para la expresión de la incertidumbre de medida y un método estocástico", del Instituto Tecnológico Metropolitano - ITM de Medellín-Colombia.

\section{REFERENCIAS}

[1] J. M. Booker and T. J. Ross, "An evolution of uncertainty assessment and quantification," Sci. Iran., vol. 18, no. 3, pp. 669-676, Jun. 2011.

[2] BIPM et al., International vocabulary of metrology-Basic and general concepts and associated terms (VIM), 3rd ed. JCGM, 2012.

[3] D. Romano, A. Bernetti, and R. De Lauretis, "Different methodologies to quantify uncertainties of air emissions," Environ. Int., vol. 30, no. 8, pp. 1099-1107, Oct. 2004.

[4] H.-J. von Martens, "Evaluation of uncertainty in measurements-problems and tools," Opt. Lasers Eng., vol. 38, no. 3-4, pp. 185-206, Sep. 2002.

[5] BIPM et al., Evaluation of measurement data - Guide to the expression of uncertainty in measurement. JCGM, 2008.

[6] BIPM et al., Evaluation of measurement data - An introduction to the "Guide to the expression of uncertainty in measurement" and related documents. JCGM, 2009.

[7] BIPM et al., Evaluation of measurement data - Supplement 2 to the "Guide to the expression of uncertainty in measurement" Extension to any number of output quantities. JCGM, 2011.

[8] K. Rypdal and K. Flugsrud, "Sensitivity analysis as a tool for systematic reductions in greenhouse gas inventory uncertainties," Environ. Sci. Policy, vol. 4, no. 2-3, pp. 117135, Apr. 2001.

[9] O. Velychko and T. Gordiyenko, "The use of guide to the expression of uncertainty in measurement for uncertainty management in National Greenhouse Gas Inventories," Int. J. Greenh. Gas Control, vol. 3, no. 4, pp. 514-517, Jul. 2009.

[10] O. N. Velichko and T. B. Gordienko, "Methods of calculating emissions of pollutants into the atmosphere and estimating their uncertainty," Meas. Tech., vol. 52, no. 2, pp. 193-199, Feb. 2009.

[11] L.-I. Tong, C.-W. Chang, S.-E. Jin, and R. Saminathan, "Quantifying uncertainty of emission estimates in National Greenhouse Gas Inventories using bootstrap confidence intervals," Atmos. Environ., vol. 56, pp. 8087, Sep. 2012.

[12] A. Ferrero and S. Salicone, "The randomfuzzy variables: A new approach to the expression of uncertainty in measurement," IEEE Trans. Instrum. Meas., vol. 53, no. 5, pp. 1370-1377, 2004.

[13] H. Cheng and A. Sandu, "Uncertainty quantification and apportionment in air quality models using the polynomial chaos method," Environ. Model. Softw., vol. 24, no. 8, pp. 917-925, Aug. 2009.

[14] J. I. DelaRosa and G. A. Fleury, "Bootstrap Methods for a Measurement Estimation Problem," IEEE Trans. Instrum. Meas., vol. 55, no. 3, pp. 820-827, Jun. 2006.

[15] I. Lira and D. Grientschnig, "Equivalence of alternative Bayesian procedures for evaluating measurement uncertainty," Metrologia, vol. 47, no. 3, pp. 334-336, 2010.

[16] Y. Hu, F. Xie, B. Wu, and Y. Wang, "An Uncertainty Quantification Method Based on Generalized Interval," in 2013 12th Mexican International Conference on Artificial Intelligence, 2013, pp. 145-150.

[17] G. Buonanno, G. Ficco, C. Liguori, and A. Pietrosanto, "The influence of the uncertainty on monitoring stack emissions in a waste-to-energy plant," in 2008 IEEE Instrumentation and Measurement Technology Conference, 2008, pp. 1771-1776.

[18] Legal Information Institute, "Environmental Protection Agency," in CFR, Legal 
Evaluación y aplicación de la incertidumbre de medición en la determinación de las emisiones de fuentes fijas: una revisión

Information Institute.

[19] Instituto de Hidrología, Meteorología y Estudios Ambientales de Colombia - IDEAM, Resolución 935. Colombia, 2011.

[20] J. F. P. Gomes, V. M. a Cruz, and M. L. C. Ribeiro, "Estimation of uncertainty in the determination of nitrogen oxides emissions," Accredit. Qual. Assur., vol. 11, no. 3, pp. 138145, May 2006.

[21] BIPM et al., Evaluation of measurement data - Supplement 1 to the "Guide to the expression of uncertainty in measurement" Propagation of distributions using a Monte Carlo method. JCGM, 2008.

[22] M. A. Azpurua, C. Tremola, and E. Paez Barrios, "Comparison of the Gum and Monte Carlo Methods for the Uncertainty Estimation in Electromagnetic Compatibility Testing," Prog. Electromagn. Res. B, vol. 34, pp. 125-144, 2011.

[23] H. Ramebäck et al., "Implementing combined uncertainty according to GUM into a commercial gamma spectrometric software," J. Radioanal. Nucl. Chem., vol. 282, no. 3, pp. 979-983, Dec. 2009.

[24] EURACHEM and CITAC, Quantifying Uncertainty in Analytical Measurements, 3rd ed. Germany, 2012.

[25] M. A. L. Traple, A. M. Saviano, F. L. Francisco, and F. R. Lourenço, "Measurement uncertainty in pharmaceutical analysis and its application," J. Pharm. Anal., vol. 4, no. 1, pp. 1-5, Feb. 2014.

[26] J. Choi, E. Hwang, H. Y. So, and B. Kim, "An uncertainty evaluation for multiple measurements by GUM," Accredit. Qual. Assur., vol. 8, no. 1, pp. 13-15, 2003.

[27] J. Choi, D. Kim, E. Hwang, and H.-Y. So, "An uncertainty evaluation for multiple measurements by GUM, II," Accredit. Qual. Assur., vol. 8, no. 5, pp. 205-2017, 2003.

[28] S. G. Rabinovich, "Accuracy of single measurements," Accredit. Qual. Assur., vol. 12, no. 8, pp. 419-424, Aug. 2007.

[29] R. Kessel, M. Berglund, and R. Wellum, "Application of consistency checking to evaluation of uncertainty in multiple replicate measurements," Accredit. Qual. Assur., vol. 13, no. 6, pp. 293-298, Jun. 2008.

[30] M. Priel, "From GUM to alternative methods for measurement uncertainty evaluation," Accredit. Qual. Assur., vol. 14, no. 5, pp. 235241, 2009.

[31] G. Nam, C.-S. Kang, H.-Y. So, and J. Choi, "An uncertainty evaluation for multiple measurements by GUM, III: using a correlation coefficient," Accredit. Qual. Assur., vol. 14, no. 1, pp. 43-47, Jan. 2009.

[32] F. Attivissimo, A. Cataldo, L. Fabbiano, and
N. Giaquinto, "Systematic errors and measurement uncertainty: An experimental approach," Measurement, vol. 44, no. 9, pp. 1781-1789, Nov. 2011.

[33] M. A. F. Martins, R. Requião, and R. A. Kalid, "Generalized expressions of second and third order for the evaluation of standard measurement uncertainty," Measurement, vol. 44, no. 9, pp. 1526-1530, Nov. 2011.

[34] A. Williams, "EURACHEM/CITAC workshop on recent developments in measurement uncertainty," Accredit. Qual. Assur., vol. 17, no. 2, pp. 111-113, Apr. 2012.

[35] P. Wei, Q. P. Yang, M. R. Salleh, and B. Jones, "Symbolic Computation for Evaluation of Measurement Uncertainty," in 2007 IEEE Instrumentation \& Measurement Technology Conference IMTC 2007, 2007, pp. $1-4$.

[36] J. M. Jurado and A. Alcázar, "A software package comparison for uncertainty measurement estimation according to GUM," Accredit. Qual. Assur., vol. 10, no. 7, pp. 373381, Jul. 2005.

[37] T. a. Zang, "On the expression of uncertainty intervals in engineering," Theor. Comput. Fluid Dyn., vol. 26, no. 5, pp. 403-414, Oct. 2012.

[38] F. Attivissimo, N. Giaquinto, and M. Savino, "A Bayesian paradox and its impact on the GUM approach to uncertainty," Measurement, vol. 45, no. 9, pp. 2194-2202, Nov. 2012.

[39] H. Imai, "Expanding needs for metrological traceability and measurement uncertainty," Measurement, vol. 46, no. 8, pp. 2942-2945, Oct. 2013.

[40] M. Thompson and S. L. R. Ellison, "Dark uncertainty," Accredit. Qual. Assur., vol. 16, no. 10 , pp. $483-487$, Oct. 2011.

[41] G. Chew and T. Walczyk, "A Monte Carlo approach for estimating measurement uncertainty using standard spreadsheet software," Anal. Bioanal. Chem., vol. 402, no. 7, pp. 2463-2469, Mar. 2012.

[42] M. Cox, P. Harris, and B. R.-L. Siebert, "Evaluation of Measurement Uncertainty Based on the Propagation of Distributions Using Monte Carlo Simulation," Meas. Tech., vol. 46, no. 9, pp. 824-833, Sep. 2003.

[43] M. Ángeles Herrador and A. G. González, "Evaluation of measurement uncertainty in analytical assays by means of Monte-Carlo simulation," Talanta, vol. 64, no. 2, pp. 415422 , Oct. 2004

[44] M. G. Cox and B. R. L. Siebert, "The use of a Monte Carlo method for evaluating uncertainty and expanded uncertainty," Metrologia, vol. 43, no. 4, pp. S178-S188, 
Evaluación y aplicación de la incertidumbre de medición en la determinación de las emisiones de fuentes fijas: una revisión

Aug. 2006.

[45] A. B. Forbes, "An MCMC algorithm based on GUM Supplement 1 for uncertainty evaluation," Measurement, vol. 45, no. 5, pp. 1188-1199, Jun. 2012.

[46] H. Janssen, "Monte-Carlo based uncertainty analysis: Sampling efficiency and sampling convergence," Reliab. Eng. Syst. Saf., vol. 109, pp. 123-132, Jan. 2013.

[47] D. a. Sheen and H. Wang, "The method of uncertainty quantification and minimization using polynomial chaos expansions," Combust. Flame, vol. 158, no. 12, pp. 23582374, Dec. 2011.

[48] E. D. Attanasi and T. C. Coburn, "A Bootstrap Approach to Computing Uncertainty in Inferred Oil and Gas Reserve Estimates," Nat. Resour. Res., vol. 13, no. 1, pp. 45-52, Mar. 2004.

[49] T. Saffaj and B. Ihssane, "A Bayesian approach for application to method validation and measurement uncertainty," Talanta, vol. 92, pp. 15-25, Apr. 2012.

[50] I. Park and R. V Grandhi, "A Bayesian statistical method for quantifying model form uncertainty and two model combination methods," Reliab. Eng. Syst. Saf., vol. 129, pp. 46-56, Sep. 2014.

[51] D. Theodorou, L. Meligotsidou, S. Karavoltsos, A. Burnetas, M. Dassenakis, and M. Scoullos, "Comparison of ISO-GUM and Monte Carlo methods for the evaluation of measurement uncertainty: Application to direct cadmium measurement in water by GFAAS," Talanta, vol. 83, no. 5, pp. 15681574, Feb. 2011.

[52] D. Theodorou, Y. Zannikou, G. Anastopoulos, and F. Zannikos, "Coverage interval estimation of the measurement of Gross Heat of Combustion of fuel by bomb calorimetry: Comparison of ISO GUM and adaptive Monte Carlo method," Thermochim. Acta, vol. 526, no. 1-2, pp. 122-129, Nov.
2011.

[53] S. Sediva and M. Havlikova, "Comparison of GUM and Monte Carlo method for evaluation measurement uncertainty of indirect measurements," in Proceedings of the 14th International Carpathian Control Conference (ICCC), 2013, pp. 325-329.

[54] A. E. Torres-Abello, "Metodología para la Estimación de Incertidumbres Asociadas a Concentraciones de Sólidos Suspendidos Totales Mediante Métodos de Generación Aleatoria," TecnoLógicas, no. 26, pp. 181200, 2011.

[55] M. G. Cox, "Propagation of distributions by a Monte Carlo method, with an application to ratio models," Eur. Phys. J. Spec. Top., vol. 172, no. 1, pp. 153-162, Jun. 2009.

[56] T. E. Lovett, F. Ponci, and A. Monti, "A Polynomial Chaos Approach to Measurement Uncertainty," IEEE Trans. Instrum. Meas., vol. 55, no. 3, pp. 729-736, Jun. 2006.

[57] R. Bun, K. Hamal, M. Gusti, and A. Bun, "Spatial GHG inventory at the regional level: accounting for uncertainty," Clim. Change, vol. 103, no. 1-2, pp. 227-244, Nov. 2010.

[58] M. Lesiv, A. Bun, and M. Jonas, "Analysis of change in relative uncertainty in GHG emissions from stationary sources for the EU 15," Clim. Change, vol. 124, no. 3, pp. 505518, Jun. 2014.

[59] K. Rypdal and W. Winiwarter, "Uncertainties in greenhouse gas emission inventories - evaluation, comparability and implications," Environ. Sci. Policy, vol. 4, no. 2-3, pp. 107-116, Apr. 2001.

[60] H. C. Frey and J. Zheng, "Quantification of Variability and Uncertainty in Emission Inventories: A Prototype Software Tool with Application to Utility NOx Emissions," Proceedings, Annu. Meet. Air Waste Manag. Assoc., p. 19, Sep. 2001. 